\title{
EEG Alpha Spindles as Indicators for Prolonged Brake Reaction Time During Auditory Secondary Tasks in a Real Road Driving Study
}

\author{
Michael Schrauf ${ }^{\mathrm{a}, \mathrm{b},{ }^{*}, \text { Andreas Sonnleitner }}{ }^{\mathrm{a}, \mathrm{c}}$, Michael Simon ${ }^{\mathrm{d}}$, Wilhelm E. Kincses ${ }^{\text {a }}$ \\ a Daimler AG, Research and Development, GR/PAP, HPC G024_BB, Hanns-Klemm-Str. 45, 71034 Böblingen, Germany \\ ${ }^{\mathrm{b}}$ University of Regensburg, Institute of Experimental Psychology, Universitätsstr. 31, 93053 Regensburg, Germany \\ ${ }^{c}$ Heinrich-Heine-University of Düsseldorf, Institute of Experimental Psychology, Universitätsstr. 1, 40225 Düsseldorf, Germany \\ ${ }^{d}$ University of Tübingen, Wilhelm-Schickard-Institut für Informatik, Sand 13, 72076 Tübingen, Germany
}

* Corresponding author at Daimler AG

\begin{abstract}
Driver distraction accounts for a substantial number of traffic accidents. Therefore, the impact of auditory secondary tasks on driving performance was examined. In addition to performance measures, i.e. reaction time on emergency brakings of a leading vehicle, mental driver states were described by electroencephalographic (EEG: alpha spindles, alpha band power) as well as cardiac activity (ECG: heart rate variability).

Results show that brake reaction time (RT) increased with time-on-task during all conditions $(\mathrm{p}<.001)$, and was significantly higher while performing the secondary task $(\mathrm{p}<.001)$. Physiological measures showed similar effects. Alpha spindle rate, alpha band power as well as heart rate variability (HRV) increased with time-on-task and were significantly different during the secondary task, indicating inhibited visual information processing and reduced concentration ability. This study shows that reduced driving performance measured by means of prolonged brake reactions during increased cognitive load elicited by auditory secondary tasks is indicated by EEG measures as well as cardiac activity, enabling the direct quantification of driver distraction in experiments during real road driving.
\end{abstract}

\section{INTRODUCTION}

A substantial number of traffic accidents can be attributed to driver distraction or inattention (Dingus et al., 2006). One possible cause are secondary tasks which distract the driver and may interfere with driving, since attention is shifted towards the secondary task (Brookhuis et al., 1991; Lamble et al., 1999). Alpha band power from the EEG is correlated with the attended modality, i.e. high alpha power during attention to auditory stimuli and lower alpha power during attention to visual stimuli (Foxe et al., 1998; Cooper et al., 2003). Further, EEG measures have proven to be suitable for the objective assessment of driver vigilance (De Waard,
1996; Lal \& Craig, 2002). Simon et al. (2011) showed that EEG alpha spindles, defined as short narrowband bursts in the alpha band, are a robust and objective measure for assessing driver vigilance under real driving conditions. The authors reported that fatigue has a rather long-term effect on the alpha spindle rate (spindle occurrence per minute), whereas short-term variations in the alpha spindle rate may indicate phasic cognitive processes (e.g. distraction from monotonous monitoring tasks like highway driving). Further, EEG alpha spindles are assumed to indicate thalamo-cortical gating for incoming sensory information (Pfurtscheller, 2003) and are therefore 
significantly involved in processing selective attention (Cohen, 1993). In addition to EEG measures, heart rate (HR) and heart rate variability (HRV) are known as suitable physiological measures for in-vehicle assessment of cognitive load (e.g. De Waard, 1996).

The study aims at investigating the shortterm effects of auditory secondary tasks on EEG measures, e.g. alpha spindle rate and alpha band power, and to identify correlates of driver distraction in cerebral and cardiac activity. In particular the short-term effects of distraction on the alpha spindle rate are of interest. It is hypothesized that auditory secondary tasks increase both alpha spindle rate and alpha band power and decrease heart rate variability. The distracting effect of the secondary task is assumed to result in slower brake reaction times in response to brake lights of a leading vehicle. Driving without secondary tasks should lead to faster reaction times as well as to a lower alpha spindle rate, lower alpha band power and higher heart rate variability.

\section{METHODS}

\section{Subjects}

20 healthy subjects ( 5 females) between 22 and 53 years of age (mean age 29.4 years) were recruited from an in-house database and were familiar with the vehicle used in this study. They had normal or correctedto-normal vision, normal hearing, reported no neurological disorders and were native speakers. Written informed consent using the HFES code of ethics was obtained prior to the study.

\section{Primary task}

The experiment took place on a non-public circuit in a disused military training area in Münsingen, Germany. The circuit had a length of $37 \mathrm{~km}$ with varying difficulties along the track due to road curvature and changes in altitude. The setup consisted of two vehicles. The leading vehicle was driven by the investigator, followed by a vehicle driven by the participant. Participants were instructed to follow the leading vehicle with a distance of approximately $20 \mathrm{~m}$. At start, the participant's vehicle was positioned $20 \mathrm{~m}$ behind the leading car to provide reference for the required safety distance. The driver upfront initiated braking maneuvers from $60 \mathrm{~km} / \mathrm{h}$ down to about $40 \mathrm{~km} / \mathrm{h}$ within an inter-stimulus interval between $42.5 \mathrm{~s}$ and $57.5 \mathrm{~s}$ which was acoustically triggered from a laptop placed inside the vehicle. The participant in the following car was instructed to react as fast as possible to any braking of the leading car (indicated by the brake lights) irrespective of the actual distance between cars. After each braking, both cars returned to the maximum speed of $60 \mathrm{~km} / \mathrm{h}$. An interlaced block design was used in which the subjects had to perform the primary driving task for three minutes followed by three minutes of one of two different secondary auditory tasks (either word detection or word detection including text content) while driving. The experiment consisted of three rounds on the circuit, adding up to a total driving duration of about two hours. This resulted in a total of 32 threeminutes-blocks (16 driving and 8 of each of the two auditory tasks), including about 150 braking events.

As reaction time we defined the time between the appearance of the braking lights of the leading vehicle and the participant's start of the braking pedal press in the following car.

\section{Auditory secondary task}

Participants listened to parts of an audio book recording ("Sieben Jahre in Tibet" [Seven Years in Tibet], Harrer, 1952). They were alternately instructed to detect the German definite article "die" (corresponding to "the" for female nouns) or the German copula "und" (engl. "and") by pressing a button fixed to their right index finger (section 1). In every threeminute-block the target word appeared between 17 and 21 times. To ensure that participants followed the secondary task continuously, the number of detected target words was counted.

In the second part of the experiment, participants had to listen to the content of the story (section 2). After each three-minutes block they were instructed to answer a question related to the content of the preceding block by choosing the correct answer out of three possible alternatives. To avoid sequential or timeon-task effects on performance, the order of the auditory tasks was balanced between subjects.

\section{Measures}

Vehicle data (i.e. speed, distance between cars, braking and acceleration) of both cars were recorded from the respective CAN-bus (Controller Area Network) and synchronized via a W-LAN connection. Subject's button presses as well as EEG and ECG data were synchronized with the 
vehicle data and stored on a notebook in the follower car.

EEG- and ECG-data were recorded with BrainProducts hard- and software (acticAP and BrainAmp amplifier). 32 EEG electrodes with $250 \mathrm{~Hz}$ sampling rate were used. Electrode placement was based on the extended international 10-20 system, using $\mathrm{FCz}$ as reference. ECG was recorded with one electrode placed at the cardiac apex. Respiration was not measured. After storage, data were re-referenced to common average, band-pass filtered from $0.5 \mathrm{~Hz}$ to $48 \mathrm{~Hz}$ and down-sampled to $128 \mathrm{~Hz}$.

\section{Preprocessing}

In order to reject remaining artifacts after online correction of ocular artifacts (online implementation according to the procedure from Gratton and Coles, 1983), an auto-regression based approach was applied (see Schlögl, 2000 for details) to identify artifact epochs which were then excluded from further analysis. Due to their proximity to muscular structures, all temporal and fronto-polar electrodes were excluded, resulting in 23 out of 32 EEG channels and one ECG channel. Alpha spindle rate was calculated in accordance to the method from simon et al. (2011). Alpha band power was calculated by computing the log power spectrum for each block and channel with Welch's modified periodogram and by subsequently averaging spectral values in the alpha band $(6-13 \mathrm{~Hz})$.

For the HRV the standard deviation for NNintervals in every three-minute-block was calculated (SDNN).

\section{Statistical analysis}

Behavioral as well as physiological data were averaged for each condition. Alpha spindle rate was calculated for each channel and subsequently averaged across all EEG channels. All measures were $z-$ transformed for each subject. Analysis of variance (ANOVA) was used for all withinsubject comparisons to identify the effect of time-on-task and distraction for each dependent measure. The level of $\alpha$ was set to .05 for all analysis. Whenever $H_{0}$ had to be rejected, the partial $\eta^{2}$ is reported as a measure of relative effect size. For time-on-task effects, a post-hoc trend analysis was calculated using polynomial contrasts.

Correlation coefficients between dependent measures were calculated for each subject and subsequently averaged over all subjects (average $\mathrm{z}$ back-transformed $r$, Silver \& Dunlap, 1987).

\section{RESULTS}

\section{Data reduction}

Since a paired samples t-test showed no significant differences between the two auditory tasks for any dependent variable (reaction time, alpha spindle rate, alpha band power, heart rate, heart rate variability), both tasks were combined into one experimental condition for further analysis. This was also justified by participants' reports according to which they paid attention to the content in both conditions, apparently not distinguishing between them.

\section{Reaction time}

Comparing driving performance for both conditions showed significantly faster brake reaction times for the condition "driving only" as compared to "driving with auditory secondary tasks"

$\left(F(1,19)=23.127, p<.001, \cdot{ }^{2}=.549\right)$.

Polynomial trend analysis revealed a significant linear increase in reaction time with ongoing time-on-task

$\left(F(1,19)=71.76, p<.001, \bullet^{2}=.791\right.$, see Fig. 1)

\section{Alpha spindles and band power}

Alpha spindle rate during the condition "driving only" was significantly lower than during "driving with auditory secondary tasks" $(F(1,19)=11.268, p<.01$, $\left.{ }^{2}=.360\right)$.

Polynomial trend analysis showed a significant quadratic increase of alpha spindle rate with time-on-task $(F(1,19)=8.749, p<.01, \bullet 2=.315)$.

"Driving with auditory secondary task" resulted in an increased alpha band power $(\mathrm{F}(1,19)=21.306, \mathrm{p}<.001, .2=.529)$ as compared to "driving only".

Trend analysis revealed no significant effect of time on alpha band power.

\section{HR and HRV}

HR constantly decreased with time-on-task (Linear: $F(1,19)=22.530, p<.001, \bullet 2=.543)$, but did not differ between the two test conditions.

Comparing HRV (SDNN) revealed a higher variability for "driving only" as compared to "driving with the auditory secondary tasks" $\left(F(1,19)=10.344, p<.01, \cdot{ }^{2}=.352\right)$. Polynomial trend analysis showed a significant linear increase in HRV with time-on-task $(F(1,19)=17.287, p<.01$, $.^{2}=.476$ ). 


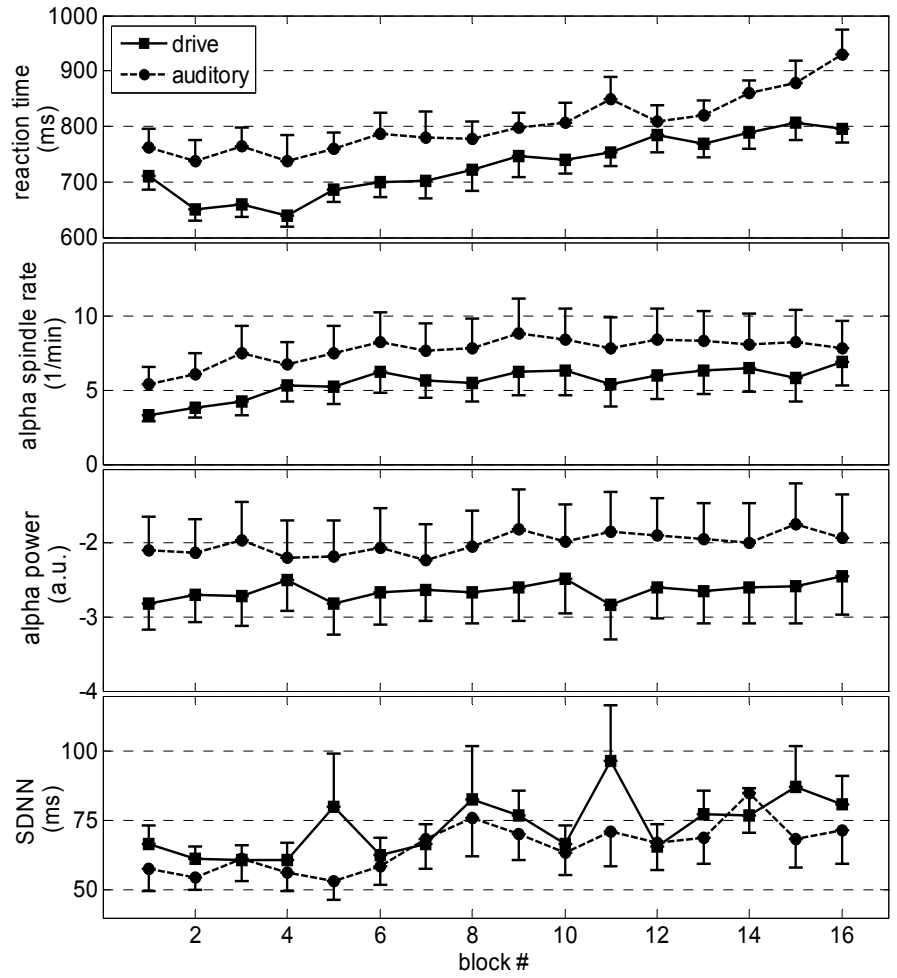

Fig. 1: Reaction time, alpha spindle rate, alpha power and heart rate variability (SDNN) for the 16 blocks is plotted separately for both conditions (black squares: "drive" (driving only), black circles: "auditory" (driving with auditory secondary tasks)). Data points represent the averaged values of $N=20$ participants. Error bars represent the standard error of the means.

\section{Correlations}

The mean correlation between alpha spindle rate and brake reaction time showed a consistent positive trend $(r=.205)$ with a confidence interval of $-.264 \cdot r \cdot .511$. Correlating alpha power and reaction time revealed a similar trend ( $r=.251$, confidence interval: -.221 • $r$ •.545). HRV and brake reaction time showed a low mean correlation $(r=.073)$ with a confidence interval of -.382 • $r .405$.

\section{DISCUSSION}

\section{Time-on-task}

In this study a strong increase of the alpha spindle rate was observed with ongoing time-on-task. Since driving is a visual task, this can be explained by a decreased processing of visual stimuli due to increasing task-related fatigue which is in line with Simon et al. (2011). In contrast, this effect was not observed for alpha band power, making this measure less sensitive to vigilance fluctuations. On the behavioral level, the vigilance decrement also resulted in an increased reaction time to brake lights.

Even though the participants were driving for only about two hours, the majority reported increased fatigue at the end of the drive. The present findings are in accordance with Simon et al. (2011) where fatigue was most strongly reflected in the alpha spindle rate during real daytime driving.

Without performing secondary tasks (driving only condition), spindle rate doubles at the end of the drive. This is accompanied by an increase of $15 \%$ for brake reaction time.

\section{Auditory secondary task}

The data clearly show that distraction leads to a degraded driving behavior represented by increased brake reaction time. Also, this attention shift towards the auditory secondary task goes along with an active inhibition of visual information processing which was evaluated by the increase in alpha spindle rate and band power. A similar response of alphaband power occurs during internalized attention (Cooper et al., 2003). Further, low levels of alpha-band activity were found to correlate with enhanced performance in visual tasks (Linkenkaer et al., 2004; van Dijk et al., 2009). This is confirmed in our study where distraction leads to a higher spindle rate during real road driving and to slower reaction times to brake lights indicating that divided attention may diminish the cognitive processing of visual information.

Task-related changes in the occurrence of alpha spindles could be observed over all cortex regions. This overall distribution fits with the assumption that alpha spindles in the awake state are controlled by interplay between thalamic relay cells and the thalamic reticular nucleus (Pfurtscheller, 2003). This allows the thalamus to provide a dynamic relay that affects the nature and format of information that reaches the cortex (Pfurtscheller \& Lopes da Silva, 1999; Sherman, 2001).

HRV values did not differ between the two auditory task conditions which is in line with findings of Tripathi et al. (2003), but were significantly lower during the secondary auditory task as compared to driving only. The lower HRV values can be interpreted as a consequence of an increased mental workload since subjects reported higher task requirements during the auditory condition at the end of the experiment. 
HR values did not show differences between any task conditions but showed a constant decrease for all subjects with time-ontask, reflecting a continuous reduction in vigilance (Schmidt et al., 2009).

Even though distraction had a similar effect on physiological and behavioural data, mean correlations were low. A mean increase from 5.55 spindles/minute during driving to 7.68 spindles/minute during the auditory secondary tasks is related to mean brake reaction times of $728 \mathrm{~ms}$ and $803 \mathrm{~ms}$ respectively. Considering the participants' awareness of the driving task and continuous braking, this increase can be considered as a high impact on internal visual processing. Pronounced individual correlations existed, but could not be found in every participant. Nevertheless, the results show that the influence of auditory secondary tasks on brake reaction times can be observed in objective measures.

\section{CONLCUSION}

The present findings emphasize the potential benefit of objectively monitoring the driver's cognitive state (i.e. EEG alpha spindle rate) during real driving conditions.

Since EEG measures are able to describe distracted driver states, the verbal assessment of the driver's mental state or it's quantification by additional tasks are no longer mandatory, enabling the design of more realistic and ecologically valid experiments.

The development and the functioning of advanced driver assistance systems will benefit from the ability to unambiguously identify periods of inattention or distraction and will help to reduce the probability of traffic accidents related to driver distraction.

\section{REFERENCES}

Brookhuis, K.A., De Vries, G. \& De Waard, D. (1991). The effects of mobile telephoning on driving performance. Accident Anal. Prev., 23, 309-316.

Cohen, R.A. (1993). The neuropsychology of attention. Critical issues in neuropsychology. New York: Plenum Press.

Cooper, N.R., Croft, R.J., Dominey, S.J.J., Burgess, A.P. \& Gruzelier, J.H. (2003). Paradox lost? Exploring the role of alpha oscillations during externally vs. internally directed attention and the implications for idling and inhibition hypotheses. Int. J. Psychophysiol., 47(1), 65-74.

De Waard, D. (1996). The measurement of drivers' mental workload. PhD thesis obtained from University of Groningen.
Dingus, T.A., Klauer, S.G., Neale, V.L., Petersen, A., Lee, S.E., Sudweeks, J., Perez, M.A., Hankey, J., Ramsey, D., Gupta, S., Bucher, C., Doerzaph, Z.R., Jermeland, J. \& Knipling, R.R. (2006). The 100-Car Naturalistic Driving Study: Phase II Results of the 100-Car Field Experiment. Interim Project Report for DTNH22-00-C-07007, Task Order 6; Report No. DOT HS 810 593). Washington, D.C.: National Highway Traffic Safety Administration.

Foxe, J.J., Simpson, G.V. \& Ahlfors, S.P. (1998). Parieto-occipital_ $10 \mathrm{~Hz}$ activity reflects anticipatory state of visual attention mechanisms. Neuroreport, 9(17), 3929-3933.

Gratton, G., Coles, M.G.H. \& Donchin, E. (1983). A new method for off-line removal of ocular artifact. Electroen. Clin. Neuro., 55(4), 468484 .

Harrer, H. \& Schwarz, M.M. (Speaker) (1952). Sieben Jahre in Tibet [CD]. Marburg: Verlag und Studio für Hörbuchproduktionen.

Lal, S.K.L. \& Craig, A. (2002). Driver fatigue: electrophysiology and psychological assessment. Psychophysiology, 39, 1-9.

Lamble, D. , Kauranen, T., Laakso, M. \& Summala, H. (1999). Cognitive load and detection thresholds in car following situations: safety implications for using mobile (cellular) telephones while driving. Accident Anal. Prev., 31(6), 617-623.

Linkenkaer-Hansen, K., Nikulin, V.V., Palva, S., Ilmoniemi, R.J. \& Palva, J.M. (2004). Prestimulus oscillations enhance psychophysical performance in humans. J. Neurosci., 24, 10186-10190.

Pfurtscheller, G. (2003). Induced Oscillations in the Alpha Band: Functional Meaning. Epilepsia, $44,2-8$.

Pfurtscheller, G. \& Lopes da Silva, F.H. (1999). Event-related EEG/MEG synchronization and desynchronization: basic principles. Clin. Neurophysiol., 110(11), 1842-1857.

Schlögl, A. (2000). The electroencephalogram and the adaptive autoregressive model: theory and applications. Aachen: Shaker Verlag

Schmidt, E.A., Schrauf, M., Simon, M., Fritzsche, M., Buchner, A. \& Kincses, W.E. (2009). Driver's midjudgement of vigilance state during prolonged monotonous daytime driving. Accident Anal. Prev., 41, 1087-1093.

Sherman, S.M. (2001). Tonic and burst firing: dual modes of thalamocortical relay. Trends Neurosci., $24(2), 122-126$

Silver, N.C. \& Dunlap W.P. (1987). Averaging Correlation Coefficients: Should Fisher's z Transformation Be Used? J. Applied Psychology, $72(1), 146-148$.

Simon, M., Schmidt, E.A., Kincses, W.E., Fritzsche, M., Bruns, A., Aufmuth, C., Bogdan, M., Rosenstiel, W. \& Schrauf, M. (2011). EEG-Alpha spindle measures as indicators of driver fatigue under real traffic conditions. Clin. Neurophysiol. 122, 1168-1178.

Tripathi, K.K., Mukundan, C.R. \& Mathew, T.L. (2003). Attentional modulation of heart rate variability (HRV) during execution of PC based cognitive tasks. Ind. J. Aerospace Med., 47(1).

Van Dijk, H., Schoffelen, J.M., Oostenveld, R. \& Jensen 0. (2008). Prestimulus oscillatory activity in the alpha band predicts visual discrimination ability. J. Neurosci., 28, 19161823 . 